\title{
The Compound Prediction Analysis of Information Network Security Situation based on Support Vector Combined with BP Neural Network Learning Algorithm
}

\author{
Jie Cheng, Bingjie Lin, Jiahui Wei, Ang Xia \\ State Grid Information \& Telecommunication Branch, Beijing 100053, China
}

Received: June 4, 2021. Revised: December 9, 2021. Accepted: January 12, 2022. Published: January 13, 2022.

\begin{abstract}
In order to solve the problem of low security of data in network transmission and inaccurate prediction of future security situation, an improved neural network learning algorithm is proposed in this paper. The algorithm makes up for the shortcomings of the standard neural network learning algorithm, eliminates the redundant data by vector support, and realizes the effective clustering of information data. In addition, the improved neural network learning algorithm uses the order of data to optimize the "end" data in the standard neural network learning algorithm, so as to improve the accuracy and computational efficiency of network security situation prediction.MATLAB simulation results show that the data processing capacity of support vector combined BP neural network is consistent with the actual security situation data requirements, the consistency can reach $98 \%$. the consistency of the security situation results can reach $99 \%$, the composite prediction time of the whole security situation is less than $25 \mathrm{~s}$, the line segment slope change can reach $2.3 \%$, and the slope change range can reach $1.2 \%$, which is better than BP neural network algorithm.

Key words-network security situation, Time series, Information network, Support vector
\end{abstract}

\section{INTRODUCTION}

$\mathrm{I}$ nformation network is an important tertiary industry activity in society, which is not only the guarantee of China's economic transformation in 2020, but also the basis for the upgrading of the tertiary industry [1]. The intelligence of information network not only expands the application scope of network, but also realizes its own structure optimization. Continuous security situation prediction and analysis of information network could help enterprise managers and government departments make decisions and improve the anti risk ability and comprehensive competitiveness of information network [2]. In the past, the prediction method of security situation in information network is mainly BP neural network algorithm. Although these algorithms could predict and analyze the complex unstable time data in network security situation, which still could not avoid the shortage of large data redundancy in MATLAB algorithm, nor could it meet the requirements of massive data calculation in network security situation. Some scholars believed that [3], the network security situation in the information network presents a big data situation, and these trends were becoming more and more obvious. The accuracy and effectiveness of traditional algorithms such as neural network, vector regression, chaotic time and neural network in network security situation prediction is decreasing day by day. It is suggested to integrate the above algorithms with redundant data elimination function. Some scholars also believed that [4], although the two times eigenvalue of the coefficient series in the time series method reduced the length of the series by $1 / 2$, which still did not have time shift invariance and could not avoid the data distortion on the right. Therefore, it is proposed to revise the steady-state and discrete functions to achieve the purpose of reconstructing the network security situation prediction results [5]. Based on the above reasons, this paper will integrates discrete support vector and neural network algorithm to improve the accuracy of the results by eliminating redundant data of network security situation, revising the right data and reconstructing the overall data, so as to better predict the network security situation.

\section{IMPROVEMENT BP NEURAL NETWORK}

\section{A. Related Concepts}

BP neural network is a quantitative and qualitative comprehensive analysis model, which realizes the interactive analysis of a large number of data. BP neural network is divided into three layers: input layer, weight layer and output layer[6]. When the input data are consistent, different weight analysis can get different results. BP neural network model can adjust the parameters of weight layer according to the results of output layer or expected requirements[7]. through the method of statistics, Artificial neural network can have simple decision-making ability and simple judgment ability like human beings. This method has more advantages than formal logical reasoning calculus. BP neural network standardizes the statistical data, finds 
meaningful eigenvalues in a large number of data, and realizes the effective analysis of data. At the same time, BP neural network uses its own local structure space to realize the integration of various data.BP neural network can realize logical analysis, interactive analysis and iterative analysis of data. It is a widely used analysis model at present [8].

\section{B. Conceptual Model of BP Neural Network}

BP model includes: input layer, enter implicit processing, and output in output layer [9]. In the process of back propagation, the threshold and weight of each device in the hidden layer will judge whether the data meets the requirements. If it meets the requirements, input or output the data, otherwise eliminate the data. The assumed sample is set as $x_{r}=\left(x_{1}, \ldots, x_{m}\right)^{T}$, each data of the hidden layer is $\alpha_{r}=\left(\alpha_{1}, \ldots, \alpha_{n}\right)^{T}$, the result of the output layer is $\mathrm{A}_{r}=\left(a_{1}, \ldots, a_{l}\right)^{T}$, and the judgment standard vector of the output result is $c_{r}=\left(c_{1}, \ldots, c_{l}\right)^{T}$. Wherein, the weights of the input layer is $w_{i j}=(i=1, \ldots, m ; j=1, \ldots, n)$ and the threshold is $b_{i j}=(i=1, \cdots, m ; j=1, \cdots, n)$ in the hidden layer; The weight between the hidden layer and the output layer is $w_{j k}=(j=1, \ldots, n ; k=1, \ldots, l)$, the threshold is $b_{j k}=(j=1, \ldots, n ; k=1, \ldots, l)$.According to the above description, the forward propagation formula of BP neural network is as follows.

$$
\left\{\begin{array}{c}
\alpha_{j}=f\left[\sum_{i=1}^{m}\left(w_{i j} x_{i}+c_{i j}\right)\right] \\
\mathrm{A}_{k}=f\left[\sum_{j=1}^{p}\left(w_{j k} \alpha_{r}+c_{j k}\right)\right]
\end{array}\right.
$$

\section{Advantages of improved BP neural network}

First, improve data processing efficiency. The improved BP neural network model adds K-means clustering analysis to cluster the original data according to the threshold, which improves the efficiency of data processing and can process massive information data [10]. The traditional BP neural network lacks the preprocessing of initial data, and the processing efficiency decreases with the increase of data volume, so it can not meet the analysis requirements of information security situation.

Second, improve the accuracy of data processing. BP neural network is improved to set the threshold of local extreme value and global extreme value, so that the processing of information security situation moves forward [11]. However, local extremum often occurs in the calculation of traditional BP neural network, which increases the error of calculation results.

\section{THE MATHEMATICAL DESCRIPTION OF COMPOUND PREDICTION OF NETWORK SECURITY SITUATION}

The key of network security situation analysis is to quantify the relevant indicators, and mathematically describe the prediction link, transmission content and transmission direction in the information network, so as to pave the way for the judgment and analysis of later prediction results [12].

\section{A. The Judgment Process of Security Situation}

Network security situation judgment includes three aspects: network structure $x_{i}$, information network impact sustainability is $x_{k}$. The network structure also includes infrastructure is $x_{i 1}$, different proportion is $x_{i 2}$, the degree of cooperation is $x_{i 3}, \ldots$; The promotion degree of security prediction to information network structure is $x_{j l}$, the integration degree of information network and security prediction is $x_{j 2}$, the promotion level of information network to security prediction is $x_{j 3}, \ldots$; The degree of cooperation among LAN, Internet and extranet $x_{k l}$, the cooperation between devices of different bridges and switches is $x_{k 2}$, the cooperation between different departments is $x_{k 3}, \ldots$. According to the above analysis, the network security situation involves many aspects, and the collected data is massive (cloud data, a large number of applications of intelligent devices) and complex (there are a large number of unstructured data), which greatly reduces the "microscopic" and "Overview" effects of the calculation results, resulting in "distortion" of the prediction. Because the mass and complexity are the inevitable trend of the development of information network, we should focus on the solution of the problem of "right data distortion".

\section{B. The Security Situation Data Flow Description}

The stable support vector comprehensively analyzes the obtained data by extracting redundant discrete, and maintains the order of data coefficients to reduce the "right data distortion" rate $[13,14]$. At the same time, the stable support vector uses discrete extraction to ensure the time shift invariance of the data and complete the single-phase feature and ordered feature extraction of the data. The specific data flow is described as follows.

Assuming that the network security situation prediction result is $A$ and $A_{l}=\left\{a_{1}, a_{2}, \ldots \ldots, a_{n}\right\}$, the relationship between $A$ and each input data is

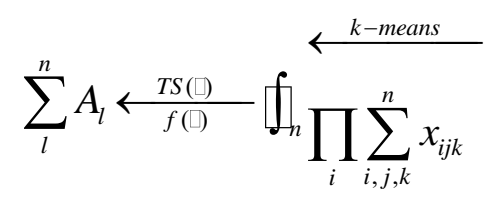

Among them, $i, j, k$ are natural numbers, BP $(\bullet)$ is the time series function, $\mathrm{f}(\bullet)$ is the stable support vector, $k$-means $(\bullet)$ is the previous data clustering function, $G(\bullet)$ is the forward function between different input indexes, and $-G(\bullet)$ is the reverse function.

Suppose that any result $a_{l}$ in the network security situation and the input $z$ in the BP neural network algorithm (information network structure is $x_{i}$, impact on the information network is $x_{j}$, and persistence of the information network is $x_{k}$ ), $P$ is the data proportion (information network structured data $>70 \%$, semi information network structured data $>70 \%$, non information network structured data $>70 \%$ ), $q$ is the data distortion processing method (reconstruction $=$ 1 , Coefficient order $=2$, quantitation $=3$, eigenvalue $=4$, 
clustering $=4)$, then $c_{l}$ is described as

$$
\begin{aligned}
& \operatorname{Inc}_{l}^{o, p, q} \\
& o \in(1,2, \ldots ., n), p \in(1,2,3,4), q \in(1,2,3,4)
\end{aligned}
$$

Among them, the logarithm In $(\bullet)$ of $c_{l}$ is used to avoid the occurrence of $|\infty|$ or extreme value 0 , so as to ensure the effectiveness of the calculation results.

For the data fusion between $x_{i}, x_{j}$ and $x_{k}$, the fusion function $f(x)$ is adopted, and the redundant data is proposed. The calculation formula of the function is expressed as follows (3).

$$
\varphi(x)=\frac{\overline{\left[\left(\sum_{i=1, j=1, k=1}^{n} \alpha_{i j k} x_{i j k}\right)+\xi\right]}}{\sum_{i, j, k=1}^{n}\left(x_{i}+x_{j}+x_{k}\right)}
$$

Among them, $\alpha_{1}, \alpha_{2}$, and $\alpha_{3}$ are the weight coefficients of information volume, information security and development trend fusion respectively, and $\xi$ is the adjustment errors of various industries. The weight coefficient is obtained from the statistical data of 2015-2020 network security situation yearbook of each department.

The data of security situation is mainly based on the Yearbook statistical data of the national IT network $[15,16]$. The calculation $t$ of security situation, the composite prediction results, and the simplification rate of composite prediction data $\iota=$ (data before clustering and $\mathrm{AC}$ processing - data after processing) / the total number of data obtained * $100 \%$. The specific formula is expressed as follows(4).

$$
\imath=\left\|\frac{H(\cdot)-G_{i, j, k}}{G_{i}+G_{j}+G_{k}}\right\| \bullet\left(\begin{array}{ccc}
\alpha_{i} & 0 & 0 \\
0 & \alpha_{j} & 0 \\
0 & 0 & \alpha_{k}
\end{array}\right) \square 00 \%
$$

Among them, $G_{i, j, k}$ is the composite prediction data of security situation of $x_{i}, X_{j}$ and $x_{k}, G_{i}, G_{j}$ and $G_{k}$ is the total amount of $x_{i}, x_{j}$ and $x_{k}$, and $H(\cdot)$ is the composite prediction data of security situation processed by clustering and algorithms, the weight of security situation data in $x_{j}$ and $x_{k}$, The specific formula is expressed as follows(5).

$$
G_{i, j, k}=\left(\begin{array}{ccc}
\alpha_{i} & 0 & 0 \\
0 & \alpha_{j} & 0 \\
0 & 0 & \alpha_{k}
\end{array}\right)
$$

In order to simplify the security situation data processing process, each weight needs to be processed to obtain the minimum value of the weight. Assuming that the initial weight is $\alpha_{0}$, the weight calculation formula of security situation data in $x_{i}, x_{j}$ and $x_{k}$ is expressed as follows(6).

$$
\alpha_{i, j, k}=\lim _{i \rightarrow \infty} f\left(i \varnothing_{j}, k\right)
$$

Among them, $\lim _{i \rightarrow \infty} f(i \varnothing j, k ; j \ell i, k ; k \varnothing i, j)$ is Fourier series.

In order to eliminate the "noise" of the transformation between information network structured, semi information network structured and non information network structured data and reduce the error of initial data acquisition, K-means clustering could be used. Because the attribute of security situation composite prediction data is relatively simple, $K$-means clustering could be carried out by Euclidean distance $[17,18]$. Firstly, set the range of information network structured, non information network structured and semi information network structured data. The formula is as follows

$$
|S|=c_{l} /\left(\sum_{l=0}^{n} c_{l}+\mu^{2}\right)
$$

Among them, $|S|$ is the Euclidean distance of each data; Security situation; $c_{l}$ is the initial cluster value; $c_{0}$ is the allowable error of clustering and $\mu$ is set by the industry in the early stage of calculation.

\section{IV.THE CONSTRUCTION OF COMPOUND PREDICTION MODEL OF SECURITY SITUATION BASED ON BP NEURAL NETWORK ALGORITHM}

\section{A. The Simplified Operator for Compound Prediction Data of Security Situation}

The compound prediction of security situation is that the digitization degree of the data obtained in the early stage, which should be $>60 \%$ before could be selected as the analysis data. Assuming that the digitization degree of data is $Q(c)$.

If the data have been taken, then the simplified formula of security situation data is expressed as follows(8).

$$
\text { ( } Q\left(c_{l}\right)<\mathrm{min}\left(S_{\text {half }}\right), c_{l} \in S_{\text {half }}
$$

If the data have been delete, then the simplified formula of security situation data is expressed as follows(9).

$$
Q\left(c_{l}\right)<\min \left(S_{\text {all }}\right), \quad c_{l} \in S_{\text {all }}
$$

If the data between take and delete,then the simplified formula of security situation data is expressed as follows(10).

$$
Q\left(c_{l}\right)<\dot{\mathrm{m} n}\left(S_{\text {non-all }}\right), \quad c_{l} \in S_{\text {non-all }}
$$

Through the above functions, the data before analysis is proposed to simplify the data processing capacity of support vector combined BP neural network.

\section{B. The Security Situation Operator}

The composite prediction of security situation considers not only the $x_{i}, x_{j}$ and $x_{k}$, but also the relationship among them. Therefore, the data relationship operator between $x_{i}$, $x_{j}$ and $x_{k}$, should be constructed. It is assumed that the relationship between the three is divided into local fusion relationship $\left(x_{i}, x_{j}\right.$ and $\left.x_{k}\right)$ and overall fusion relationship, which are expressed by $P c$ and $P m$ respectively. The calculation formula of overall extreme value is expressed as follows(11). 


$$
P_{\mathrm{c}}\left\{\begin{array}{cr}
P_{c_{0}}=1 & P_{\mathrm{c}}=P_{m} \\
\frac{\varphi(x)}{\left(P_{c_{x_{i}, j, k}}-P_{m}\right)}\left[\left(P_{c_{x, j, j}}-P_{c_{x i}}\right)\right] P_{c} \neq P_{m}
\end{array}\right.
$$

The calculation formula of location extreme value is expressed as follows(12).

$$
P_{\mathrm{m}}\left\{\begin{array}{cc}
P_{c_{0}} \cdot P_{c_{x_{i, j}, k}} & P_{m} \approx 1 \\
\left.\varphi(x) \overline{\left[\left(P_{c_{x_{i, j}, k}}-P_{\min }\right)\right.} \cdot P_{\text {max }}\right], P_{\text {max }}<1
\end{array}\right.
$$

Among them, $P_{c_{0}}$ is local fusion, all fusion, $P_{\min }$ and $P_{\max }$ are the minimum and maximum values of $P c$ and $P m$ fusion respectively. This value is set by the IT industry association and $\varphi(x)$ is the fusion degree function of the above analysis.

\section{The Accuracy Operator of Compound Prediction of Security Situation}

Accuracy is the key index of compound prediction of security situation [19], and it is also the main purpose of support vector combined BP neural network optimization. Therefore, the accuracy operator should be constructed.

If $0<\operatorname{Inc} c_{l}^{o, p, q}<1$, then The calculation formula is expressed as follows(13).

$$
\operatorname{Inc} c_{l}^{o, p, q}=n c_{l}^{o, p, q}
$$

If $I n c_{l}^{o, p, q}>1$, then the calculation formula as follows(14).

$$
\operatorname{Inc} c_{l}^{o, p, q}=\int_{I, j}^{n} \min \left(\operatorname{Inc} c_{l}^{o, p, q}\right)
$$

If $\operatorname{Inc}_{l}^{o, p, q}<0$, then the calculation formula as follows(15).

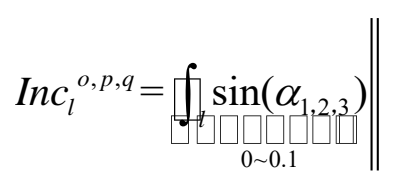

Because of the accuracy of $\operatorname{Inc} c_{l}^{o, p, q}$ is the accuracy of $x_{i}$, $x_{j}$ and $x_{k}$ data, so $0<\operatorname{Inc_{l}}{ }^{o, p, q}<1$ is required to shorten the range of calculation accuracy. At the same time, the fusion error of the calculation results should be reduced, so the specific formula is expressed as follows(16).

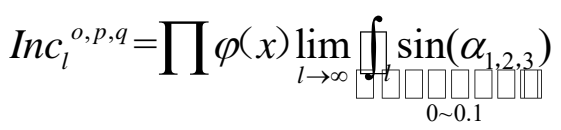

\section{The Calculation Steps of Each Operator}

According to the construction of the above operators, the calculation steps of the IT industry security situation composite prediction model could be obtained, as follows:

Build a security situation set, $C_{l}=\left\{c_{1}, c_{2}, \ldots \ldots, c_{i}\right\}$, and eliminate security situation data with digitization degree < $60 \%$. Determine the weight coefficient and adjustment error according to the Yearbook of network security situation of IT industry from 2018 to 2020 ;

The data $c_{l}$ after $K$-means clustering is processed with information network structure, semi information network structure and non information network structure, so as to realize the "cleaning" of security situation data.

Judge the fusion relationship of the "cleaned" data, and obtain the values of $P c$ and $P m$ of local and overall fusion relationships.

Carry out iterative analysis on steps $1 \sim 4$ with MATLAB software, and output the accuracy, calculation time and effectiveness of calculation results.

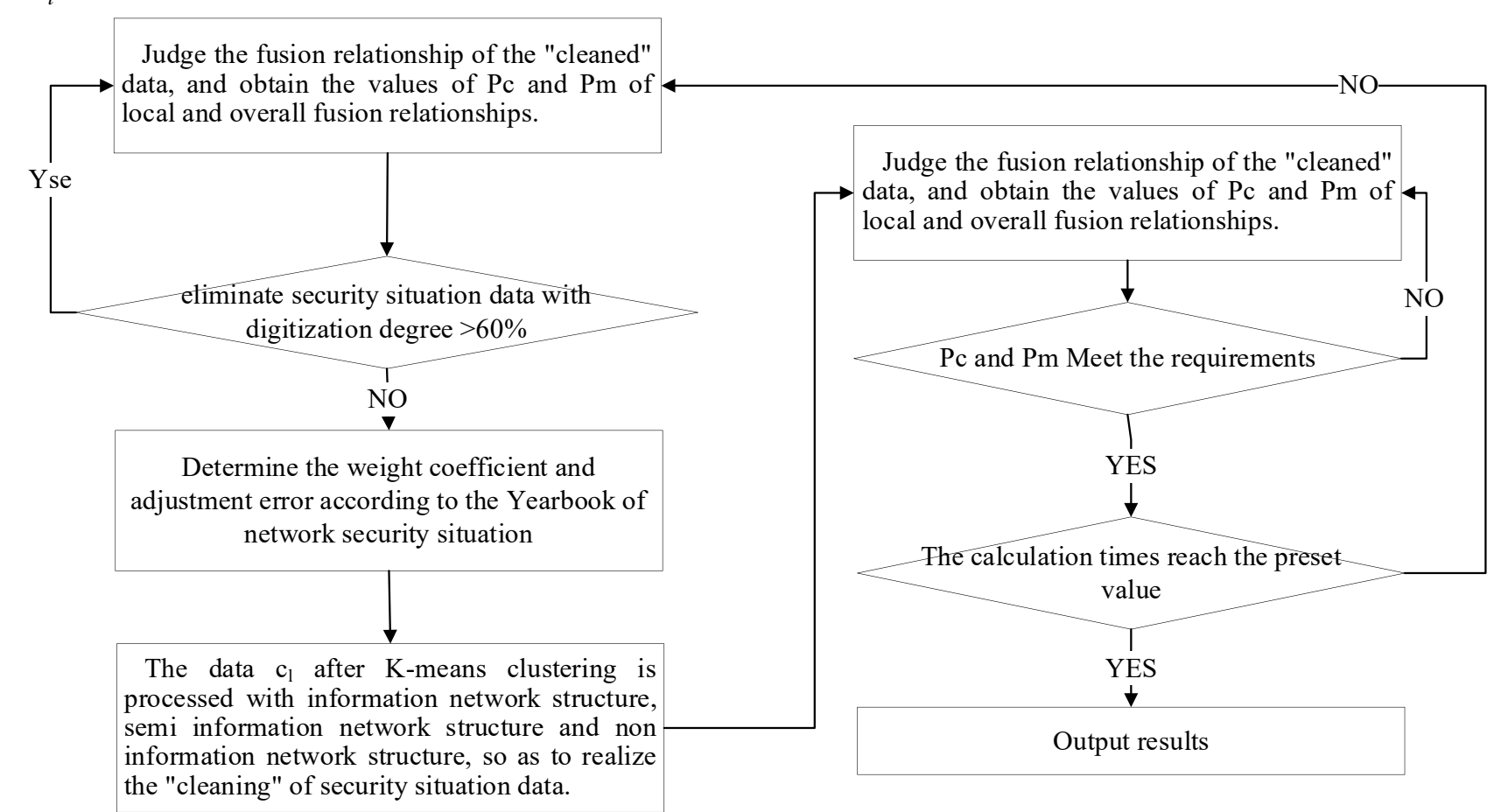

Figure 1. The flow chart 
V. THE VERIFICATION OF COMPOUND PREDICTION MODEL BASED ON SUPPORT VECTOR COMBINED BP NEURAL NETWORK

\section{A. Actual Case Analysis}

The 150 text transmitted in $302 \sim 306$ servers of company B from January to June 2020 is taken as the research object to analyze the network security situation. 150 iterative training shall be conducted for all data in the transmitted text.16 clients in the company transmit files through the above 4 servers, and calculate the data transmission volume and data loss between the sender and the receiver. At the same time, according to the log information in the server, judge the accuracy of the early warning results sent by each client.Taking $x_{i}, x_{j}$ and $x_{k}$ as input indicators, $\xi$ is the adjustment errors of various industries, $c_{0}$ is the allowable error of clustering and $\mu$ is set by the industry in the early stage of calculation, $\alpha_{0}$ is the initial weight, $P m$ is overall fusion relationship. The specific values of the above indicators are shown in Table.1.

Table 1. Initial data

\begin{tabular}{llll}
\hline parameter & numerical value & parameter & numerical value \\
\hline$c_{0}$ & 12.3 & $\mu$ & 0.03 \\
iterations & 150 & $\alpha_{0}$ & 0.21 \\
$p_{\text {min }}$ & 0.23 & $p_{\text {max }}$ & 0.83 \\
\hline
\end{tabular}

Data sources are shown in Table 2.

Table 2. Data source of security situation

\begin{tabular}{lll}
\hline Ascription & Collection type & Collect content \\
\hline The server & It network, information, big data & Enterprise: network loss and \\
LAN & Personal information, company information, overall information & information; Error correction, \\
Network group & It network, encrypted documents, files, general files, internal files & user access, etc; In terms of \\
Private server & Send, accept, lose & users: satisfaction, complaint \\
Network cloud & Send, accept, lose & rate, etc; \\
Internet of things & network, encrypted documents, files, general files, internal files & In terms of network, it involves: \\
big data & network, encrypted documents, files, general files, accept, lose & digital platform construction \\
& & [20] \\
\hline
\end{tabular}

B.The Comparison of Processing Results of Security Situation Data between Support Vectors Combined BP Neural Network Algorithm and BP Neural Network Algorithm

Support vector combined with BP neural network uses
K-mean clustering to preprocess safety situation data, eliminate redundant data and extract eigenvalues. The processing result is better than BP neural network algorithm. The result is shown in Figure 2.

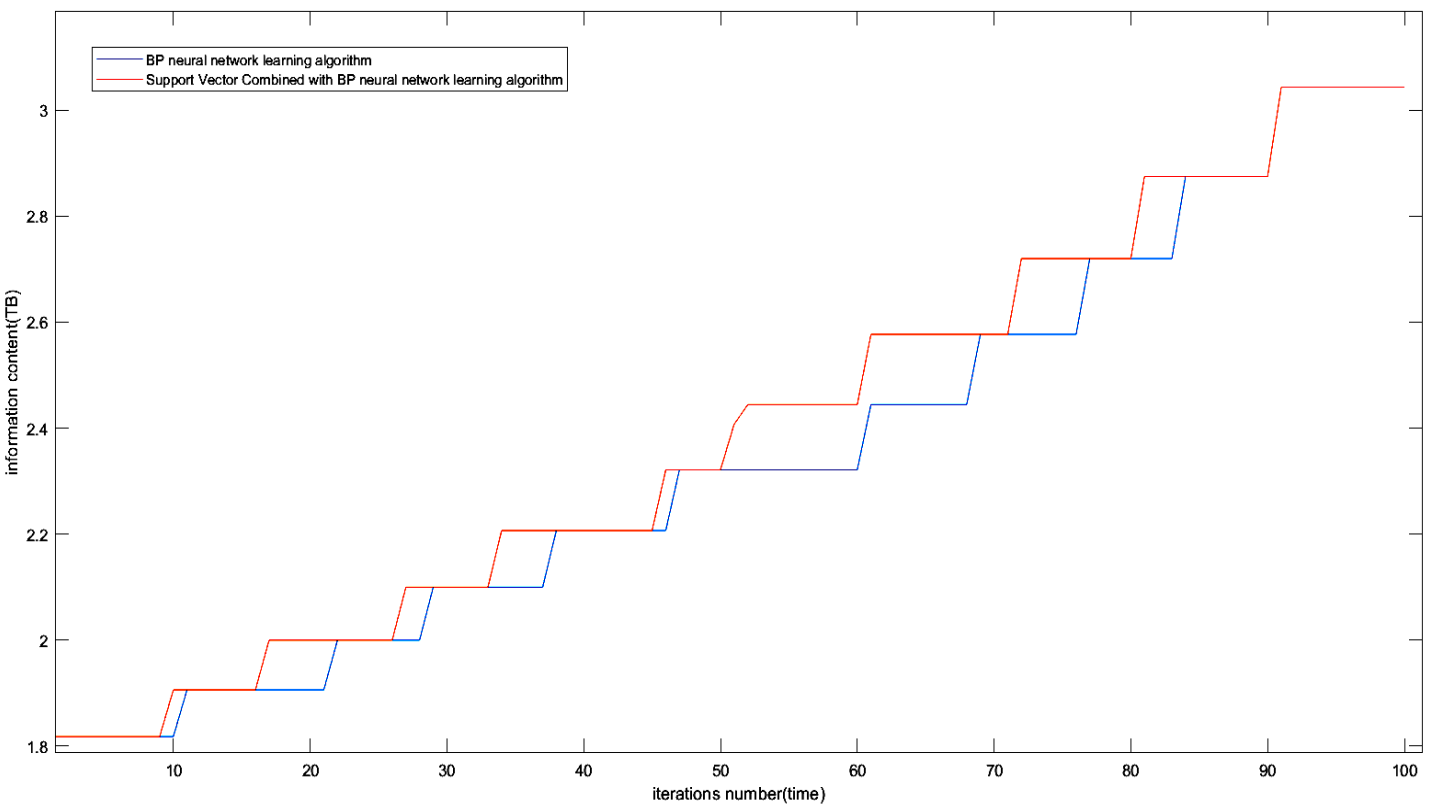

Figure 2. The Comparison of data processing results

It could be seen from Figure 1 that the data processing capacity of support vector combined BP neural network is consistent with the actual security situation data requirements, the consistency can reach $98 \%$. while the data processing capacity of BP neural network is poor consistent with the security situation, the consistency only can reach $90 \%$, and does not change along the change of the security situation line, especially at the positions of 28, 45, 58, 75, 78 and 98 iterations. This shows that the support vector combined BP neural network algorithm meets the 
requirements in the processing capacity of security situation data, and is better than BP neural network algorithm. algorithm is used to analyze the composite prediction results of security situation, and the results are shown in Figure 3.

C. Calculation accuracy of Support Vector Combined with BP Neural Network for Security Situation Data

Support vector combined with BP neural network

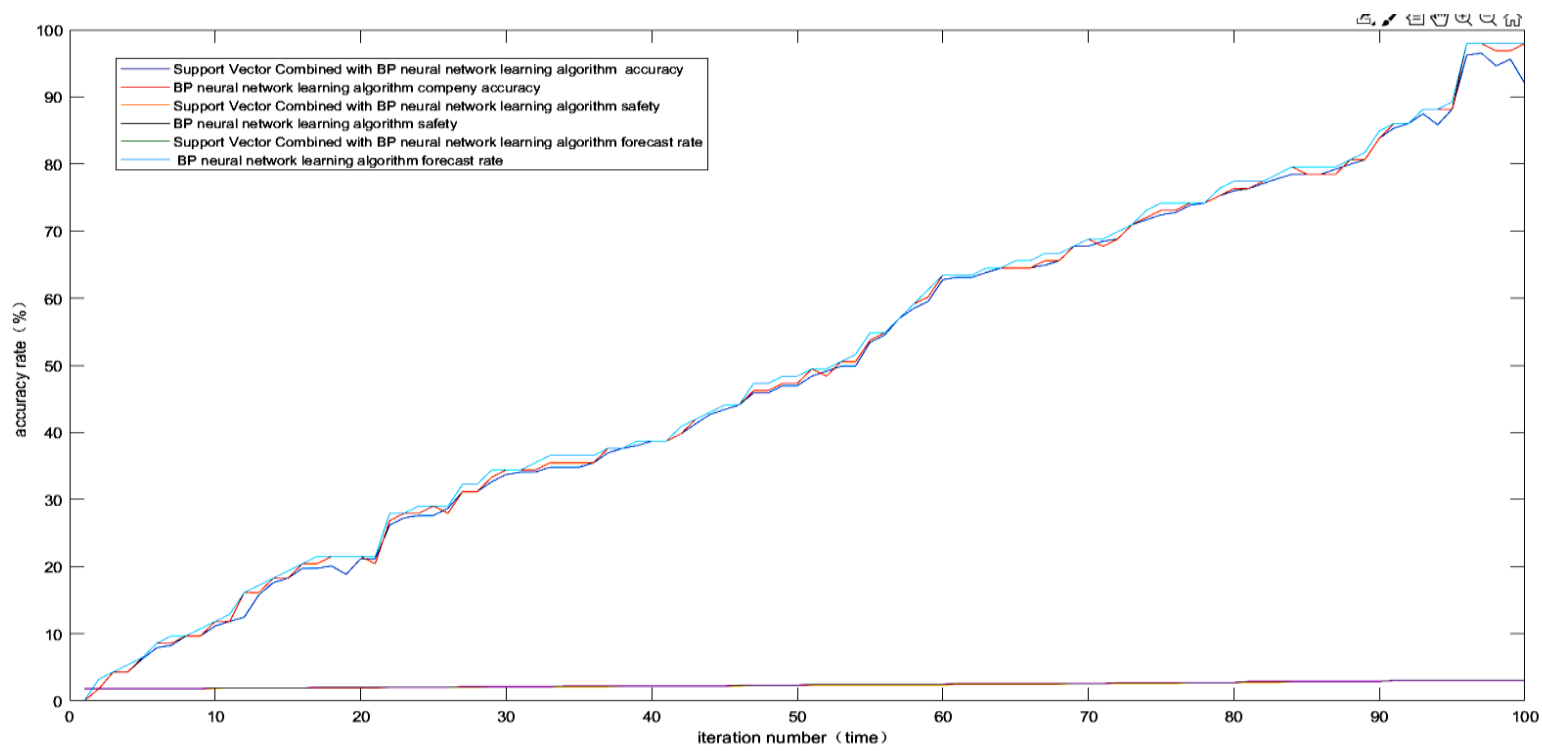

Figure 3. The Composite prediction results of security situation

The calculation accuracy can be judged by comparing the curve fitting degree of different algorithms.It can be seen from Figure 3 that the processing results of support vector combined BP neural network algorithm in the composite prediction of security situation are basically consistent with the security situation results of the actual investigation, the consistency can reach $99 \%$. while the security situation results of BP neural network is poor consistent with the security situation, the consistency only can reach $95 \%$. Among them, the results of customers are 54 and 95 iterations, and enterprises have small errors at 24, 73 and 88 iterations, which is due to the incomplete collection of security situation data of support vector combined BP neural network.

\section{Time of Compound Prediction of Security Situation}

Time is another indicator of support vector combined BP neural network algorithm. The time of compound prediction of security situation is shown in Figure 4.

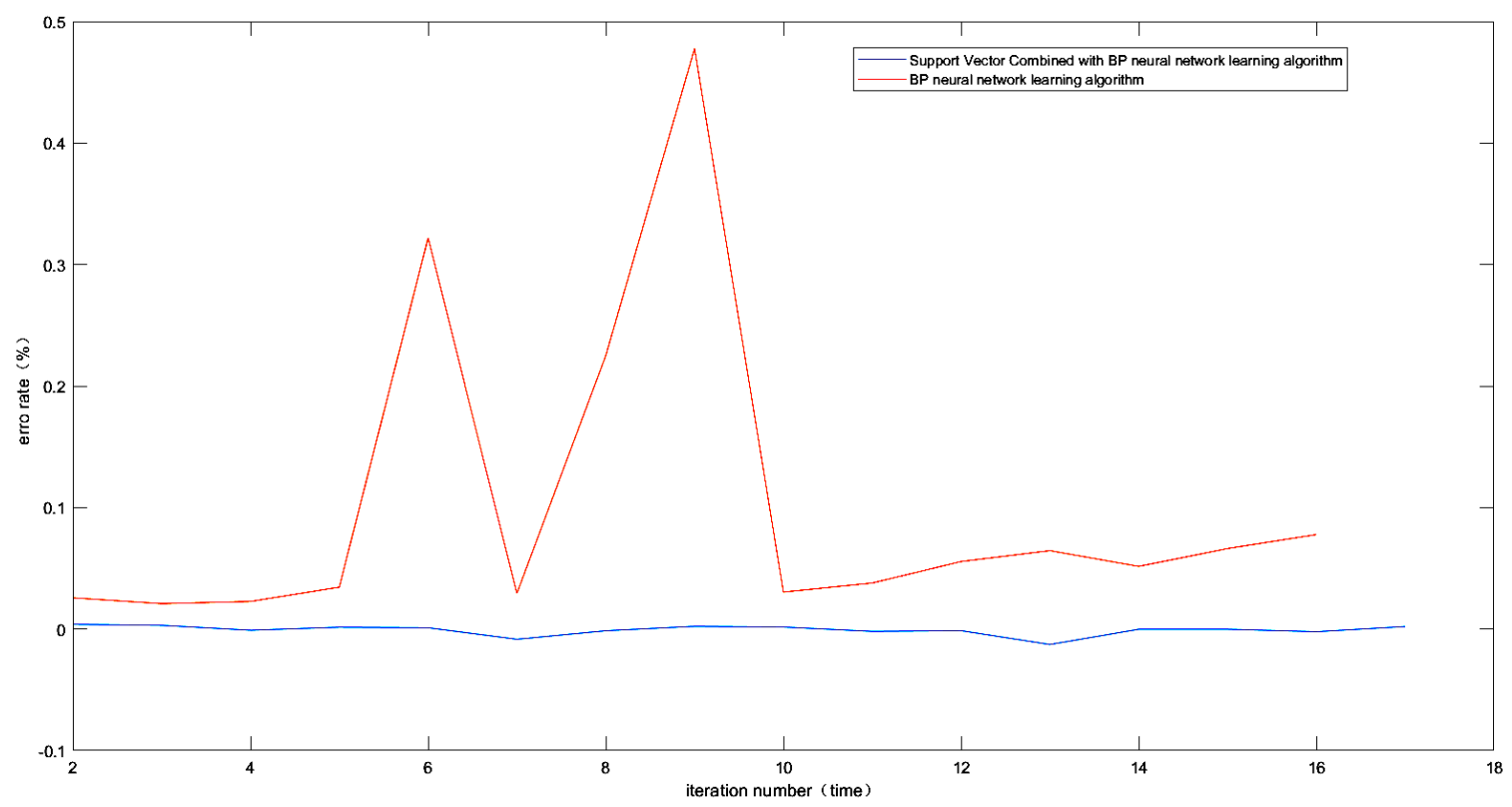

Figure 4. The security situation processing time

It can be seen from Figure 4 that the security situation processing time of support vector combined BP neural network is significantly less than that of BP neural network, and the composite prediction time of the whole security situation is less than 25s. According to the line segment slope of the two algorithms, the security situation processing of BP neural network shows ups and downs, while the line segment slope change of support vector combined BP neural 
network can reach $2.3 \%$, and the slope change range can reach $1.2 \%$. while the line segment slope change of BP neural network can reach $30 \%$ and the slope change range can reach $12 \%$. This shows that the support vector combined BP neural network algorithm is better than BP neural network algorithm, and can meet the requirements of compound prediction of security situation.

\section{CONCLUSION}

Digital economy is the requirement of the national IT network and the Ministry of industry and information technology for the development of IT industry in 2020, and it is also the direction of future development and reform of IT industry. Since 2010, the IT industry has gradually strengthened the security situation[21]. However, due to the large amount of data involved in the security situation involving customers, enterprises and information networks, the previous BP neural network, Bayesian and genetic algorithm have achieved accurate composite prediction, which affects the implementation of later improvement measures [21]. In this paper, cluster analysis and BP neural network method are combined to build a support vector combined BP neural network algorithm model to make compound prediction of security situation. The results show that: 1) the model can effectively eliminate redundant data and accurately extract eigenvalues, which is consistent with the actual safety situation evaluation results, and the calculation time is less than $25 \mathrm{~s}$, and the compound prediction process is stable; 2) Compared with the mainstream algorithm BP neural network for security situation compound prediction, the constructed model is superior to BP neural network in terms of calculation accuracy, data processing capacity and calculation time, and meets the requirements for security situation evaluation in the Ministry of industry and information technology, it industry and it summit in 2020 ; 3) Compared with the traditional BP neural network model, the improved BP neural network model can process massive data, and the accuracy of the processing results is higher. With a large increase of intelligent equipment in the future, the improved BP neural network model can meet the prediction of information security situation, which has important theoretical guiding significance for information security monitoring.

Although the redundant data is eliminated and the data processing efficiency is improved, the research on data continuity is not deep enough. In the future research process, we should strengthen the continuity research in information security situation prediction.

\section{Reference}

[1] Chen Wenjing. Research on association rules of it economic development based on Apriori algorithm. Science and technology and economy guide. 2019, 27 (24): 4-5.

[2] Yin Shuo, Liu Junhui, Bai Hongkun, et al. Research and judgment on the development trend of it economy in the process of crossing the middle-income trap - evidence based on Henan. It big data. 2019, 22 (03): 56-65.

[3] Chen Hongwei, Wang Wancheng, Wang Jituo. Application of hybrid adaptive particle swarm optimization algorithm in it economic scheduling. Computer and modernization. 2019 (03): 45-50.

[4] Luo Yan. Power enterprises going to sea: conveying development momentum -- an exclusive interview with Li Yanbin, Dean of School of economics and management of North China it University. Minsheng weekly. 2019 (17): 32-33.

[5] He kejing. Research on legal solutions to economic disputes between power enterprises in the new era. Legal system Expo. 2019 (10): 144-152.

[6] Sun miaoping, Jiang Bo. Design of distributed it economic scheduling algorithm considering communication delay. Control theory and application. 2020, 37 (11): 2303-2311.

[7] Zhang Dawei. Discussion on it system economic operation and it economic dispatching. Digital world. 2020 (07): 285.

[8] Zhang Qiuyan, song Qiang, Zhang Junwei, et al. A new method of it economic feature extraction based on extended panel big data. IT network and cleaning. 2021, 37 (02): 64-70.

[9] Zhang Chunyun, Zou dexuan, Shen Xin. Application of improved particle swarm optimization algorithm in it economic scheduling. Manufacturing automation. 2021, 43 (01): 53-57.

[10] Network security situation analysis group of CCID think tank. Development prospect of China's network security situation in 2021: China Computer News. 20213.

[11] Wen Hua. Multi party collaborative construction of "double Gigabit" network, enriching applications and promoting the development of network security situation. Communication world. 2021 (08): 26-27.

[12] Hsu C, Quangthanh N, Chien F, et al. Evaluating green innovation and performance of financial development: mediating concerns of environmental regulation. Environmental science and pollution research international. 2021.

[13] Ching-Chi H, Ngo Q, Fengsheng C, et al. Evaluating green innovation and performance of financial development: mediating concerns of environmental regulation. Environmental Science and Pollution Research. 2021(prepublish).

[14] BP neural network o $\mathrm{x}$, Zhang $\mathrm{R}$, Chen D, et al. Performance investigation and multi objective optimization of helibp neural network 1 baffle heat exchangers based on thermal and economic analyses. International Journal of heat and mass transfer. 2021, 176.

[15] Shi Chenhao, Tang Zhong, Dai Weiyang, et al. Multi objective optimization strategy of active information network based on multi-element improved NSGA II algorithm. Power supply and consumption. 2021, 38 (06): 50-55.

[16] Lin Shan, Wang Hong, Qi Linhai, et al. Short term load forecasting based on conditional generation countermeasure network. IT system automation. 2021, 45 (11): 52-60.

[17] Meng Qingyan, Wang Jingjing. Improvement and case study of partition picking method based on Support Vector Combined BP neural network. Electronic design engineering. 2021, 29 (11): 44-49. 
[18] Sun Ruijuan, Liang Jun, Wang Kewen, et al. Research review of offshore wind power collection system. IT construction. 2021, 42 (06): 105-115.

[19]Roumen Trifonov, Slavcho Manolov, Georgi Tsochev, Galya Pavlova, Automation of Cyber Security Incident Handling through Artificial Intelligence Methods, WSEAS Transactions on Computers.2019,18(2): 274-280.

[20]Arti Vaish, Anand Sharma, Anshu Sharma, Review Report on User Authentication Methods in Cyber Security, WSEAS Transactions on Communications. 2020, 19(6): 149-154.

[21]K.Thamizhmaran, IOT Supported Security Considerations for Network, WSEAS Transactions on Communications. 2020, 19(7):113-123.

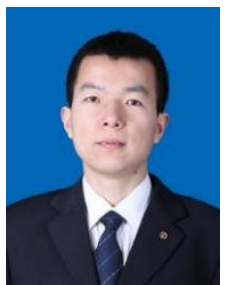

Jie Cheng, graduated from Beijing University of Posts and Telecommunications (BUPT) with a master's degree in Computer Technology and Application in 2007 , is currently working in State Grid Information \& Communication Branch as a senior engineer of the Network Security Monitoring Center. His main research directions is Information Security.

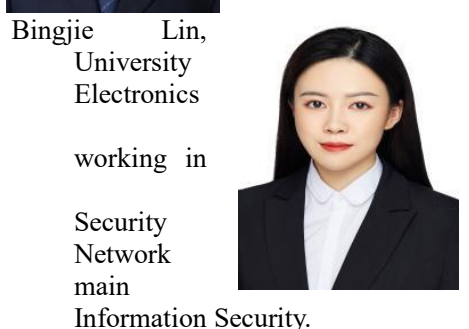

graduated from Peking
with a master's degree in
and Communication
Engineering in 2019, is currently
State Grid Information \&
Communication Branch as the
Analysis specialist of the
Security Monitoring Center. Her
research directions is

Information Security.

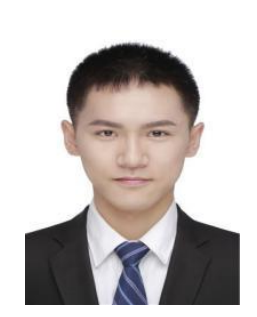

Jiahui Wei, graduated from North China Electric Power University with a master's degree in Computer Application Technology in 2018, is currently working in State Grid Information \& Communication Branch as the Security Analysis specialist of the Security Monitoring Division of the Network Security Monitoring Center. His main research directions is Information Security.

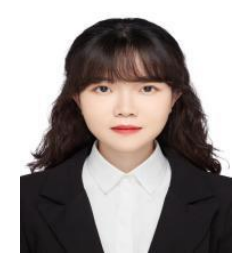

Ang Xia, graduated from North China Electric Power University with a master's degree in Information and Communication Engineering in 2020, is currently working in State Grid Information \& Telecommunication Branch as the assistant of the Internet Security Monitoring Center. Her main research directions are network information security and theory of information and communication.

\section{Creative Commons Attribution License 4.0}

\section{(Attribution 4.0 International, CC BY 4.0)}

This article is published under the terms of the Creative

Commons Attribution License 4.0

https://creativecommons.org/licenses/by/4.0/deed.en_US 